\title{
In vitro synergistic activity of amikacin combined with subinhibitory concentration of tigecycline against extended spectrum $\beta$-lactamase-producing Klebsiella pneumoniae
}

\author{
Elsayed Aboulmagd ${ }^{1,2 \star}$, Abdulrahman A. Alsultan ${ }^{1}$, Hamdan I. Al Mohammad ${ }^{1}$, Soad Al-Badry \\ and Eman M. Hussein ${ }^{1}$ \\ ${ }^{1}$ Department of Biomedical Sciences, Division of Medical Microbiology, College of Medicine, King Faisal University, \\ Kingdom of Saudi Arabia. \\ ${ }^{2}$ Department of Microbiology, Faculty of Pharmacy, Alexandria University, Alexandria, Egypt.
}

Accepted April 23, 2013

\begin{abstract}
The number of extended-spectrum $\beta$-lactamases-producing Klebsiella pneumoniae (ESBL-KP) strains is constantly increasing. This is of great concern in human healthcare around the world. In this study, we aimed to evaluate the antimicrobial activity of amikacin-tigecycline combination against six tigecyclineresistant ESBL-KP clinical isolates using disc diffusion method, checkerboard titration assay and timekill curve technique. Presence of subinhibitory concentration of tigecycline $(1 \mathrm{mg} / \mathrm{L})$ enhanced the susceptibility of ESBL-KP isolates to amikacin expressed by the percentage of relative inhibition zone diameter (\% RIZD) which ranged from 110 to $167 \%$. The fractional inhibitory concentration indices (FICls) of amikacin-tigecycline combinations were from 0.31 to 0.75 . For KP 125 and KP 135 isolates, combination of tigecycline $(2 \mathrm{mg} / \mathrm{L})$ and amikacin $(8 \mathrm{mg} / \mathrm{L})$ resulted in $99.99 \%$ killing after $24 \mathrm{~h}$ incubation. Drastic and rapid bactericidal effect was shown after $6 \mathrm{~h}$ incubation against KP 135 in the presence $2 \mathrm{mg} / \mathrm{L}$ tigecycline plus $16 \mathrm{mg} / \mathrm{L}$ amikacin. In conclusion, the combined effect of amikacintigecycline is significantly synergistic at concentrations that were within the clinically achievable serum levels and may be eligible for further evaluation in vivo against ESBL-KP infections to define their utility as an alternative to carbapenems.
\end{abstract}

Key words: ESBL, Klebsiella pneumoniae, amikacin, tigecycline, checkerboard, Time-kill curve.

\section{INTRODUCTION}

Antibiotic resistance is a serious problem in clinical medicine. The efficacy of treatment with the widely use $\beta$ lactam antibiotics is constantly challenged by the emergence of new resistant bacterial strains. In Gramnegative bacteria, $\beta$-lactamase production is considered the main antibiotic resistant mechanism (Livermore, 2009).

Extended spectrum beta-lactamase (ESBL) production is one of the main mechanisms of resistance to $\beta$-lactam antibiotics among the family Enterobacteriaceae (Szabó et al., 2001). ESBL-producing Enterobacteriaceae are among the most important and frequent nosocomial pathogens and are also resistant to many classes of antibiotics (O'Neill, 2008; Vidaillac et al., 2009). Since the 1980s, there have been numerous reports from different healthcare settings worldwide on outbreaks 
caused by ESBL-producing Klebsiella pneumoniae "ESBL-KP" (Arpin et al., 2003; Keynan and Rubinstein, 2007)

Treatment of serious life-threatening infections due to ESBL-KP pathogens presents a difficult challenge due to limited therapeutic options (Shanthi and Sekar, 2011). Combination therapy may play a crucial role in eradicating ESBL-KP and is considered one of the best options available to treat infections caused by such pathogens (Dawis et al., 2003; Klibanov et al., 2004). In addition, the use of combination therapy can broaden the spectrum of antibacterial activity, minimize the emergence of resistant bacteria and can result in synergic interaction (Dawis et al., 2003).

Tigecycline is the first glycylcycline antibiotic available for clinical use (Pankey, 2005; Seputienè et al., 2010). It is active against a wide range of clinically important Gram-negative pathogens (Hawkey and Finch, 2007). Some clinical isolates tend to have decreased susceptibility to tigecycline e.g. Proteus mirabilis, Klebsiella pneumoniae and Pseudomonas aeruginosa (Souli et al., 2006; Kresken et al., 2009). Limited data are available on combination of amikacin and tigecycline against ESBL- KP.

The objective of the present work was to evaluate the in vitro activity of amikacin-tigecycline combination against ESBL-KP by a three-step approach, involving preliminary disc diffusion method, checkerboard technique and time-kill assay.

\section{MATRERIALS AND METHODS}

\section{Bacterial isolates and antimicrobial agents}

A total of 37 non-duplicate ESBL-producing K. pneumoniae clinical isolates were collected from three hospitals in Al-Ahsa, eastern region of Saudi Arabia. VITEK 2 compact automated system (BioMerieux, Marcy l'Etoile, France) was used for identification of the isolates and testing of the antibiotic sensitivity using GN (REF. 21341) and AST-N116 (REF. 22289) kits, respectively, according to the instructions of the manufacturer. Amikacin and tigecycline were purchased from Sigma, Aldrich (St. Louis, MO, USA). All antimicrobial assays were carried out in Mueller-Hinton broth (MHB, Oxoid Ltd, UK). Antibiotic discs were purchased from Oxoid Ltd, UK.

Determination of the minimum inhibitory concentration of tigecycline and amikacin

The minimum inhibitory concentrations (MICs) of amikacin and tigecycline against six tigecycline-resistant selected clinical isolates (KP 101, 102, 111, 121, 125 and 135) were determined by broth microdilution technique using 96-well plates according to Clinical and Laboratory Standards Institute (CLSI, 2008) guidelines. The experiments were carried out in duplicate. The MIC was defined as the lowest concentration at which there was no visible bacterial growth after incubation at $37^{\circ} \mathrm{C}$ for 18 to $24 \mathrm{~h}$.

\section{Disc diffusion assay}

The effect of subinhibitory concentration of tigecycline $(1 \mathrm{mg} / \mathrm{L})$ on the antibacterial activity of amikacin was assessed by determination of the percentage of relative inhibition zone diameter (\% RIZD) using agar diffusion method according to a previously published report (Aboulmagd et al., 2011).

\section{Checkerboard titration method}

The combination between amikacin and tigecycline was assessed by checkerboard titration method (Pillai et al., 2005) in 96-well microtiter plate (Greiner bio-one $\mathrm{GmbH}$, Frickenhausen, Germany). The final bacterial inoculum was adjusted at about $10^{6} \mathrm{CFU} / \mathrm{ml}$. The experiments were carried out in duplicate. The fractional inhibitory concentration index $(\mathrm{FICl})$ for each combination was calculated using the following formulae: $\mathrm{FICl}=$ ( $\mathrm{MIC}$ of amikacin in combination $\div$ MIC of amikacin alone) plus (MIC of tigecycline in combination $\div \mathrm{MIC}$ of tigecycline alone). The $\mathrm{FICl} \leq 0.5$ indicated synergy while $\mathrm{FICl}$ of $>0.5$ but $<1$ indicated partial synergy. The additive effect was indicated by $\mathrm{FICl}$ of 1.0 while $\mathrm{FICl}$ of $>1$ but $<4$ was considered as indifferent. $\mathrm{FICl}$ of $\geq 4$ was interpreted as antagonistic (Dawis et al., 2003).

\section{Time-kill curve analysis}

Combined bactericidal effects of amikacin and tigecycline were evaluated in duplicate against KP 125 and 135 using time-kill assay (Pillai et al., 2005). Initial inoculums of about $10^{6} \mathrm{CFU} / \mathrm{ml}$ of the two isolates were exposed to tigecycline $(2 \mathrm{mg} / \mathrm{L})$ and amikacin $(2,4,8$ or $16 \mathrm{mg} / \mathrm{L}$ ) either alone or in combination. Suitable controls lacking amikacin and tigecycline were included in the experiment. Aliquots of bacterial cultures were plated onto Mueller Hinton agar at $0 \mathrm{~h}$ and after 3, 6 and $24 \mathrm{~h}$. Colonies were counted after $24 \mathrm{~h}$ incubation at $37^{\circ} \mathrm{C}$. The experiments were carried out in duplicate. The rate of killing was determined by plotting the log number of survivors per $\mathrm{ml}$ (log CFU/mL) against the time. Synergy was defined as $a \geq 2 \log _{10}$ decrease in CFU/mL by the drug combination when compared with the most active one after $24 \mathrm{~h}$.

\section{RESULTS}

The resistance patterns of 37 non-duplicate ESBLproducing Klebsiella pneumoniae clinical isolates were determined against 20 antimicrobial agents by VITEK 2 compact automated system (data not shown). All isolates were susceptible to imipenem and meropenem while tigecycline was inactive against six isolates (KP 101, 102, $111,121,125$ and 135). The resistance pattern of the six tigecycline-resistant isolates is depicted in Table 1 and these isolates were used further to evaluate the efficacy of amikacin-tigecycline combination by checkerboard technique and time-kill assay. The minimum inhibitory concentration (MIC) values summarized in Table 2 show that KP 125 and KP 135 isolates were resistant to amikacin (32 and $64 \mathrm{mg} / \mathrm{L}$, respectively) while the other four tested isolates (KP 101, 102, 111 and 121) were susceptible (MIC values $\leq 8 \mathrm{mg} / \mathrm{L}$ ).

Figure 1 shows that presence of subinhibitory concentration of tigecycline $(1 \mathrm{mg} / \mathrm{L}=0.062-0.125 \times \mathrm{MIC})$ enhanced the susceptibility of the six tested tigecyclineresistant isolates to amikacin as expressed by percentage relative inhibition zone diameter (\% RIZD). 
Table 1. Antibiotic Resistance Patterns of Six Selected ESBL Klebsiella pneumoniae Isolates.

\begin{tabular}{ll}
\hline Isolate code & Antibiotic resistance pattern $^{a}$ \\
\hline KP 101 & AMP, SAM, PIP, CFZ, CXM, CAE, FOX, CPD, CTX, GEN, TOB CIP, LEV, TGC, SXT \\
KP 102 & AMP, SAM, PIP, TZP, CFZ, CXM, CAE, FOX, CPD, CTX, CAZ, FEP, GEN, TOB, CIP, TGC, SXT \\
KP 111 & AMP, SAM, PIP, TZP, CFZ, CXM, CAE, FOX, CPD, CTX, CAZ, FEP, GEN, TOB, CIP, LEV, TGC, SXT \\
KP 121 & AMP, PIP, CFZ, CXM, CAE, CPD, CTX, GEN, TOB, CIP, LEV, TGC \\
KP 125 & AMP, PIP, CFZ, CXM, CAE, FOX, CPD, CAZ, GEN, TOB, AMK, TGC, SXT \\
KP 135 & AMP, SAM, PIP, TZP, CFZ, CXM, CAE, FOX, CPD, CTX, CAZ, FEP, GEN, TOB, AMK, CIP, LEV, TGC, SXT \\
\hline
\end{tabular}

${ }^{a}$ The abbreviations of the antibiotic discs used are: AMP, ampicillin; SAM, ampicillin/sulbactam; PIP, piperacillin; TZP, piperacillin/tazobactam; CFZ, Cefazolin; CXM, cefuroxime; CAE, cefuroxime axetil; FOX, cefoxitin; CPD, cefpodoxime; CTX, cefotaxime; CAZ, ceftazidime; FEP, cefepime; GEN, gentamicin; TOB, tobramycin; AMK, amikacin; CIP, ciprofloxacin; LEV, levofloxacin; TGC, tigecycline; SXT, sulphamethoxazol/trimethoprim.

Table 2. Minimum inhibitory concentrations (MICs) of tigecycline and amikacin against six ESBL Klebsiella pneumoniae Isolates.

\begin{tabular}{lcc}
\hline \multirow{2}{*}{ Isolate code } & \multicolumn{2}{c}{ MIC (mg/L) } \\
\cline { 2 - 3 } & Tigecycline & Amikacin \\
\hline KP 101 & 16 & 4 \\
KP 102 & 16 & 8 \\
KP 111 & 8 & 4 \\
KP 121 & 8 & 8 \\
KP 125 & 8 & 32 \\
KP 135 & 8 & 64 \\
\hline
\end{tabular}

The highest effect was recorded against KP 125 and KP 135 (167 and 150\% RIZD, respectively) while KP 101 was the least affected isolate (Figure 1).

Fractional inhibitory concentration indices (FICls) of amikacin-tigecycline combinations against the six tigecycline-resistant selected isolates exhibited synergic effect $(\mathrm{FICl} \leq 0.5)$ against $\mathrm{KP} 102,111,125$ and 135 while partial synergy $(\mathrm{FICl}>0.5-0.75)$ was observed against KP 101 and 121 (Table 3). No antagonism was observed during this study.

The bactericidal activity of amikacin-tigecycline combination was evaluated by time-kill assay and the results are shown in Figures 2 and 3 . Combination of 2 $\mathrm{mg} / \mathrm{L}$ tigecycline and $4 \mathrm{mg} / \mathrm{L}$ amikacin showed 4.8 and $6.4 \log _{10}$ reduction of number of survivors after $24 \mathrm{~h}$ incubation against KP 125 and 135, respectively (Figure 2). On the other hand, addition of $2 \mathrm{mg} / \mathrm{L}$ tigecycline to 16 $\mathrm{mg} / \mathrm{L}$ amikacin showed $99.99 \%$ killing after 6 and $24 \mathrm{~h}$ incubation against KP 135 and 125, respectively, as shown in Figure 3.

10

\section{DISCUSSION}

Infections caused by ESBLs pose serious challenge to clinicians because they are resistant to a broad range of $\beta$-lactams including $3^{\text {rd }}$ generation cephalosporins
(Ramphal and Ambrose, 2006). Moreover, ESBL-KP is recognized as one of the most common causes of healthcare associated bacterial infections together with Escherichia coli and Pseudomonas aeruginosa (Goyal et al., 2009).

Due to the limited number of effective antimicrobial agents or novel therapeutic strategies, multi-drug resistant gram-negative pathogens currently represent a serious clinical concern (Arpin et al., 2003). In the present study, the outcome of amikacin-tigecycline interactions was investigated using checkerboard titration method and time-kill curve analysis.

All tested ESBL-KP isolates were susceptible to imipenem and meropenem. However, overuse of carbapenem can lead to emergence of carbapenemresistant ESBL-KP. Therefore, to avoid the emergence of such resistant pathogens, clinicians are encouraged to seek out for other alternatives for treatment of infections caused by ESBL-KP. Combination therapy is often beneficial for patients with serious infections caused by ESBL-KP. In addition to the improvement of the killing effect of the drugs, combination therapy may also reduce the emergence of resistance and improve the spectrum of activity (Bouza and Muñoz, 2000).

Checkerboard titration assay $(\mathrm{FICl})$ and percentage relative inhibition zone diameter (\%RIZD) showed significant synergism between amikacin and tigecycline. Checkerboard analysis showed that amikacin is synergic or partially synergic in combination with tigecycline against ESBL-KP. None of the combinations resulted in antagonism. These findings are in agreement with that of Vouillamoz et al. (2008) who showed that amikacintigecycline combination exhibited synergism against $40 \%$ of ESBL-KP tested. Moreover, Petersen et al. (2006) showed that the combination of tigecycline with amikacin demonstrated synergy against $56 \%$ of the strains tested. In addition, combination studies of gentamicin plus tigecycline resulted in synergy against $20 \%$ of ESBL-KP tested (Cha, 2008).

In order to confirm the results of synergism obtained by checkerboard technique $(\mathrm{FICl} \leq 0.5)$, time-kill kinetic experiments were performed. Amikacin-tigecycline 
Table 3. Fractional inhibitory concentration index (FICl) of tigecycline-amikacin combinations against six ESBL Klebsiella pneumoniae Isolates.

\begin{tabular}{lcc}
\hline Isolate code & FICl & Effect of combination \\
\hline KP 101 & 0.75 & $\mathrm{PS}^{a}$ \\
KP 102 & 0.50 & $\mathrm{~S}^{b}$ \\
KP 111 & 0.50 & $\mathrm{~S}$ \\
KP 121 & 0.75 & $\mathrm{PS}$ \\
KP 125 & 0.31 & $\mathrm{~S}$ \\
KP 135 & 0.31 & $\mathrm{~S}$ \\
\hline
\end{tabular}

${ }^{a}$ PS, Partial synergy; ${ }^{b}$ S, Synergy.

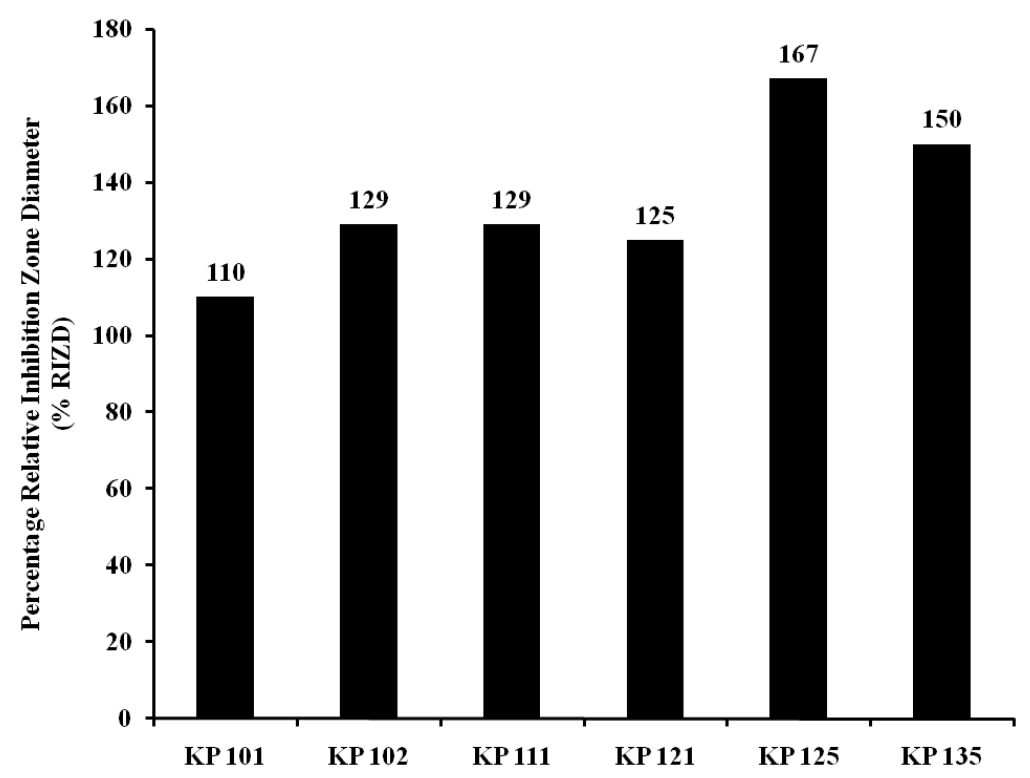

Figure 1. Effect of subinhibitory concentration of tigecycline $(1 \mathrm{mg} / \mathrm{L})$ on the susceptibility of six tigecycline-resistant isolates to amikacin expressed as percentage relative inhibition zone diameter (\%RIZD).
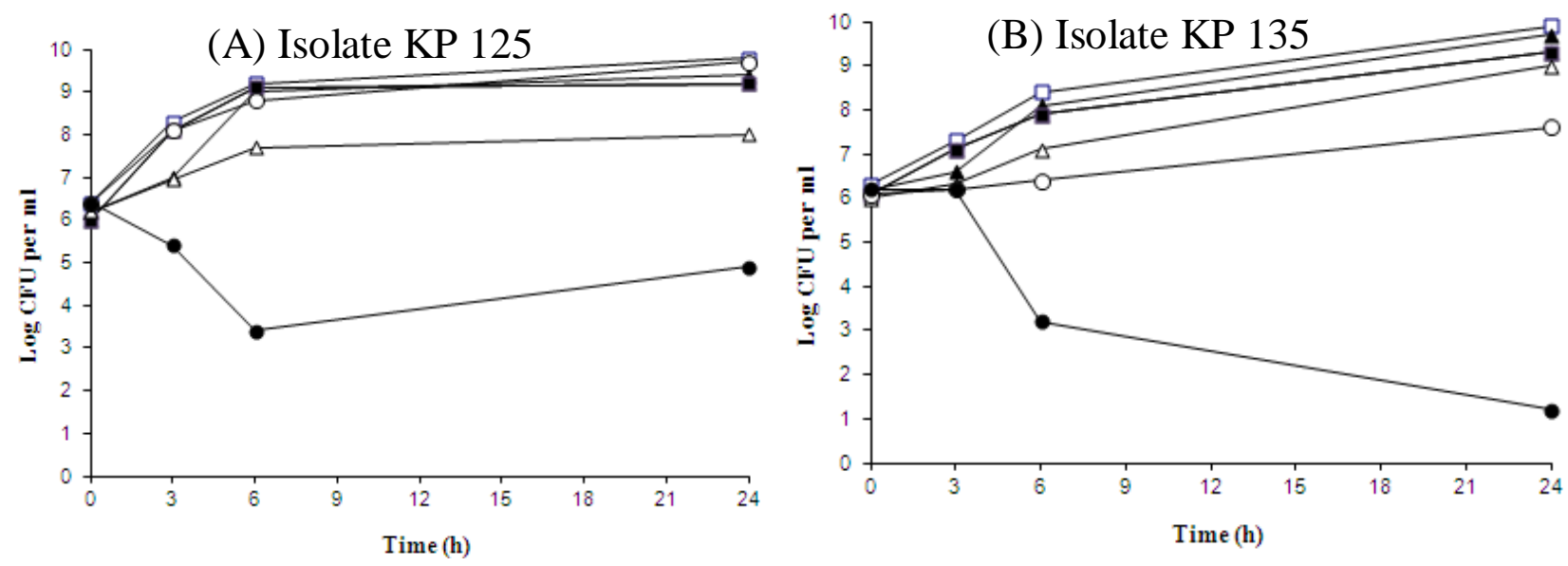

Figure 2. Time-kill studies of amikacin and tigecycline alone and in combination against (A) KP 125 and (B) KP 135 . $\square$,

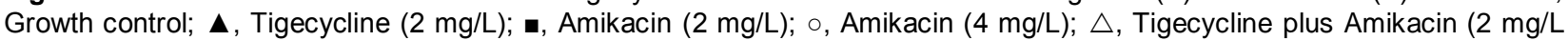
each); •, Tigecycline (2 mg/L) plus Amikacin (4 mg/L). 

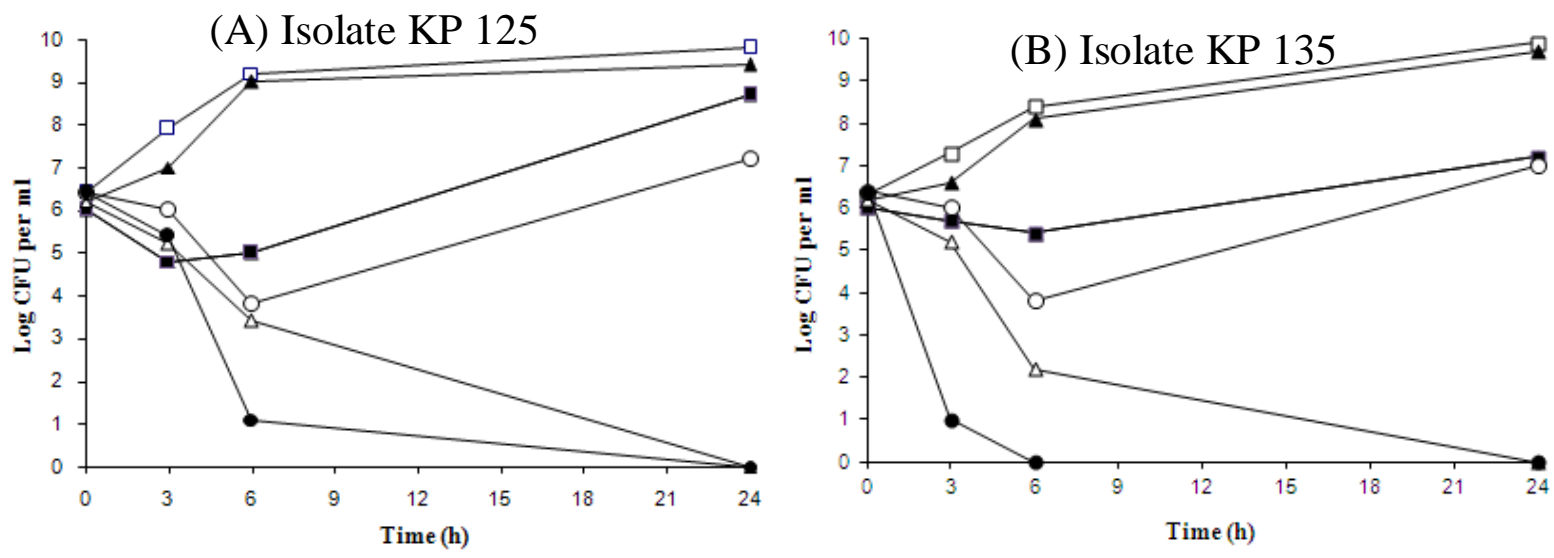

Figure 3. Time-kill studies of amikacin and tigecycline alone and in combination against two isolates: (A) KP 125 and (B) KP 135. 口, Growth control; $\mathbf{\Delta}$, Tigecycline (2 mg/L); $\mathbf{a}$, Amikacin (8 mg/L), ○, Amikacin (16 mg/L); $\triangle$, Tigecycline (2 mg/L) plus Amikacin (8 mg/L); •, Tigecycline (2 mg/L) plus Amikacin (16 mg/L).

combination revealed bactericidal synergy against the two tested ESBL-KP. These results appeared consistent with those presented by Vouillamoz et al. (2008) who showed that amikacin-tigecycline combination exhibited bacteriostatic synergism against many of the gram negative pathogens tested.

The results of the present study demonstrate that timekill analyses were in accordance with checkerboard results. In contrast, previously published data recorded certain type of discrepancy between the checkerboard and the time-kill results during studying the combination of tigecycline with different antimicrobial agents (Petersen et al., 2006; Aliskan et al., 2009).

Based on our findings, further studies of amikacintigecycline combinations, including in vivo studies in infected animal models, are required to confirm the potential utility of amikacin-tigecycline combination to combat ESBL-KP infections.

In conclusion, amikacin-tigecycline combination showed a drastic bactericidal effect at concentrations that were within the clinically achievable serum levels. This combination represents a promising therapeutic option to overcome ESBL-KP infections. Therefore, further studies are warranted to elucidate the clinical value of this combination against ESBL-KP and to define their utility as an alternative to carbapenems.

\section{REFERENCES}

Aboulmagd E, Al-Mohammad HI, AL-Badry S (2011). Synergism and postantibiotic effect of green tea extract and imipenem against methicillin-resistant Staphylococcus aureus. Microbiol. J. 1(3):89-96.

Aliskan H, Can F, Demirbilek M, Colakoglu S, Kilic S, Arslan H (2009). Determining in vitro synergistic activities of tigecycline with several other antibiotics against Brucella melitensis using checkerboard and time-kill assays. J. Chemother. 21(1):24-30.

Arpin C, Dubois V, Coulange L, André C, Fischer I, Noury P, Grobost F, Brochet JP, Jullin J, Dutilh B, Larribet G, Lagrange I, Quentin C (2003). Extended-spectrum beta-lactamase-producing
Enterobacteriaceae in community and private health care centers. Antimicrob. Agents Chemother. 47(11): 3506-3514.

Bouza E, Muñoz P (2000). Monotherapy versus combination therapy for bacterial infections. Med. Clin. North. Am. 84(6):1357-1389.

Cha R (2008). In vitro activity of cefepime, imipenem, tigecycline, and gentamicin, alone and in combination, against extended-spectrum beta-lactamase-producing Klebsiella pneumoniae and Escherichia coli. Pharmacotherapy 28(3):295-300.

Clinical and Laboratory Standards Institute "CLSI" (2008). Methods for dilution antimicrobial susceptibility tests for bacteria that grow aerobically; approved standard, 8th ed. Clinical and Laboratory Standards Institute, Wayne, PA.

Dawis MA, Isenberg HD, France KA, Jenkins SG (2003). In vitro activity of gatifloxacin alone and in combination with cefepime, meropenem, piperacillin and gentamicin against multidrug-resistant organisms. J. Antimicrob. Chemother. 51:1203-1211.

Goyal A, Prasad KN, Prasad A, Gupta S, Ghoshal U, Ayyagari A (2009). Extended spectrum beta-lactamases in Escherichia coli and Klebsiella pneumoniae and associated risk factors. Indian J. Med. Res. 129(6):695-700.

Hawkey P, Finch R (2007). Tigecycline: in-vitro performance as a predictor of clinical efficacy. Clin. Microbiol. Infect. 13(4):354-362.

Keynan Y, Rubinstein E (2007). The changing face of Klebsiella pneumoniae infections in the community. Int. J. Antimicrob. Agents 30(5):385-389.

Klibanov OM, Raasch RH, Rublein JC (2004). Single versus combined antibiotic therapy for gram-negative infections. Ann. Pharmacother. 38(2):332-337.

Kresken M, Leitner E, Brauers J, Geiss HK, Halle E, von Eiff C, Peter G, Seifert H (2009). Susceptibility of common aerobic pathogens to tigecycline: Results of a surveillance study in Germany. Eur. J. Clin. Microbiol. Infect. Dis. 28:83-90.

Livermore DM (2009). Beta-Lactamases- the Threat Renews. Curr Protein. Pept. Sci. 10(5):397-400.

O'Neill AJ (2008). New antibacterial agents for treating infections caused by multi-drug resistant gram-negative bacteria. Expert Opin. Investig. Drugs 17:297-302.

Pankey GA (2005). Tigecycline. J. Antimicrob. Chemother. 56(3):470480.

Petersen PJ, Labthavikul P, Jones CH, Bradford PA (2006). In vitro antibacterial activities of tigecycline in combination with other antimicrobial agents determined by chequerboard and time-kill kinetic analysis. J. Antimicrob. Chemother. 57:573-576.

Pillai SK, Moellering RC, Eliopoulos GM (2005). Antimicrobial combinations. In: Antibiotics in Laboratory Medicine, Ed. V. Lorian, 5th ed. Lippincott Williams \& Wilkins. pp. 365-440.

Ramphal R, Ambrose PG (2006). Extended-spectrum beta-lactamases and clinical outcomes: current data. Clin. Infect. Dis. Suppl. 4:S164- 
S172.

Šeputienè V, Povilonis J, Armalytė J, Sužiedèlis K, Pavilonis A, Sužiedèlienè $E$ (2010). Tigecycline - how powerful is it in the fight against antibiotic-resistant bacteria? Medicina (Kaunas) 46(4):240248.

Shanthi M, Sekar U (2011). In vitro activity of tigecycline against gram positive and gram negative isolates in a tertiary care hospital. J. Clin. Diag. Res. 5(8):1559-1563.

Souli M, Kontopidou FV, Koratzanis E, Antoniadou A, Giannitsioti E, Evangelopoulou P, Kannavaki S, Giamarellou H (2006). In vitro activity of tigecycline against multiple-drug-resistant, including panresistant, gram-negative and gram-positive clinical isolates from Greek hospitals. Antimicrob. Agents Chemother. 50(9):3166-3169.

Szabó D, Máthé A, Filetóth Z, Anderlik P, Rókusz L, Rozgonyi F (2001). In vitro and in vivo activities of amikacin, cefepime, amikacin plus cefepime, and imipenem against an SHV-5 extended-spectrum betalactamase-producing Klebsiella pneumoniae strain. Antimicrob. Agents Chemother. 45(4):1287-1291.
Vidaillac C, Leonard SN, Sader HS, Jones RN, Rybak MJ (2009). In vitro activity of ceftaroline alone and in combination against clinical isolates of resistant gram-negative pathogens, including betalactamase-producing Enterobacteriaceae and Pseudomonas aeruginosa. Antimicrob. Agents Chemother. 53(6):2360-2366.

Vouillamoz J, Moreillon P, Giddey M, Entenza JM (2008). In vitro activities of tigecycline combined with other antimicrobials against multiresistant gram-positive and gram-negative pathogens. J. Antimicrob. Chemother. 61(2):371-374. 